\title{
Intrahepatic portal occlusion by microspheres: a new model of portal hypertension in the rat
}

\author{
V Jaffe, B Alexander, R T Mathie
}

\begin{abstract}
Available experimental models of portal hypertension are based either on cirrhosis or externally applied portal vein constricting devices. A new method is described of raising portal pressure, which uses intraportally injected microspheres to block intrahepatic portal radicles, which has the advantages of retaining normal liver architecture and providing a more clinically relevant intrahepatic obstruction to portal flow. Measured aliquots of microspheres $(15,25,50,90 \mu \mathrm{m})$ or equivalent volumes of saline were injected into a peripheral portal tributary (caecal vein) of 22 normal rats. The resultant changes in arterial, portal, and splenic pulp pressures were monitored. Sequential microsphere injections produced graduated rises in portal pressure up to a peak of $18.5-22.5 \mathrm{~mm} \mathrm{Hg}(8 \cdot 7-12.4 \mathrm{~mm} \mathrm{Hg}$ increase from basal), which declined gradually to a steady state pressure of $13 \cdot 3-15 \cdot 1 \mathrm{~mm} \mathrm{Hg}$ (4.0-5.0 $\mathrm{mm} \mathrm{Hg}$ increase). There was no significant difference between pressure increases produced by microspheres of differing sizes. It is concluded that portal hypertension can be produced acutely by blocking portal radicles with microspheres. The maximum pressure achieved, however, is substantially less than that obtained by total portal vein occlusion (mean: $57.6 \mathrm{~mm} \mathrm{Hg}$ ). This suggests the existence of functional intrahepatic portal systemic shunts not previously described in the normal liver.
\end{abstract}

(Gut 1994; 35: 815-818)

The management of portal hypertension (PHT) has for long periods rested almost exclusively on the reduction in flow in variceal vessels, either surgically or endoscopically. Recently, however, it has become apparent that PHT is more a systemic disorder with, in particular, a hyperdynamic splanchnic circulation. ${ }^{1}$ Efforts have therefore been directed at the pharmacological manipulation of the gastrointestinal and hepatic circulations to reduce splanchnic and extrahepatic variceal flow ${ }^{2}$ while maintaining or even increasing hepatic portal inflow through the use of intrahepatic portal vasodilators. ${ }^{3}$

Research into pharmacological or surgical manipulation of portal flow has been frustrated

TABLEI Intraportallyinjected aliquotsof microspheres

\begin{tabular}{llllll}
\hline Sphere diameter $(\mu \mathrm{m})$ & No of animals & No of aliquots & $\begin{array}{l}\text { Aliquot volume } \\
(\mathrm{ml})\end{array}$ & $\begin{array}{l}\text { No of spheres/ } \\
\text { aliquot }\end{array}$ & $\begin{array}{l}\text { Total no } \\
\text { of spheres }\end{array}$ \\
\hline 15 & 6 & 10 & $0 \cdot 15$ & $5 \times 10^{5}$ & $5 \times 10^{6}$ \\
25 & 5 & 10 & $0 \cdot 2$ & $1 \times 10^{5}$ & $1 \times 10^{6}$ \\
50 & 5 & 10 & $0 \cdot 2$ & $5 \times 10^{4}$ & $5 \times 10^{5}$ \\
90 & 6 & 10 & $0 \cdot 2$ & $1 \times 10^{3}$ & $1 \times 10^{4}$ \\
\hline
\end{tabular}

by the lack of a reliable, reproducible, and clinically comparable animal model of PHT. Prehepatic portal occlusion and cirrhotogens have been used, but their use has been limited, in the first by the rapid development of portoportal collaterals, which decompress the portal occlusion, and in the second by the variability of the liver damage induced. They thus both fail to fulfil the above criteria and, in particular, do not permit the study of subtle changes in the intra and extrahepatic portal circulation.

There are two strands of evidence to suggest that microspheres could be utilised to create an alternative model of PHT, by occluding intrahepatic portal radicles. Firstly, it has been shown that over $95 \%$ of intraportally infused $15 \mu \mathrm{m}$ microspheres lodge in the normal liver. ${ }^{+6}$ Secondly, exogenously applied pancreatic cells, when injected intraportally, have been shown to produce PHT. ${ }^{7}$ It therefore seemed possible that microspheres could be used to occlude the intrahepatic portal tract, thus creating a model of PHT that could be rapidly and reliably established, and would be fairly comparable with the clinical situation. Furthermore, in contrast with prehepatic portal occlusion models, the intrahepatic nature of this block would seem to preclude the formation of decompressing portoportal collaterals.

The aims of this acute experimental study were: (1) to confirm that microspheres given intraportally would produce an increase in portal pressure; (2) to measure the number and diameter of microspheres required to induce such a pressure increase; and (3) to evaluate the maximum pressure attainable.

\section{Methods}

Twenty two adult male Wistar rats $(300-400 \mathrm{~g}$ : MRC, Mill Hill) were anaesthetised with ether and cannulae placed in the right carotid artery and in the caecal vein. A $21 \mathrm{G}$ butterfly was inserted into the pulp of a mobilised spleen. Pressure transducers recorded systemic arterial, portal venous, and splenic pulp pressures (Fig 1). When steady resting pressures had been achieved for at least five minutes, 10 sequential aliquots of microspheres of varying diameters (see Table I) were manually injected through the portal cannula over a 20 minute period, each injection taking 10-15 seconds to accomplish. Equivalent volumes of saline were injected into control animals during the same time course. Changes in portal, splenic, and arterial pressures were monitored - in particular, the peak pressures induced by intraportal injections were recorded. Animals were then observed for a further 20-60 minutes until a new steady state had been reached. After this period a soft clamp was 


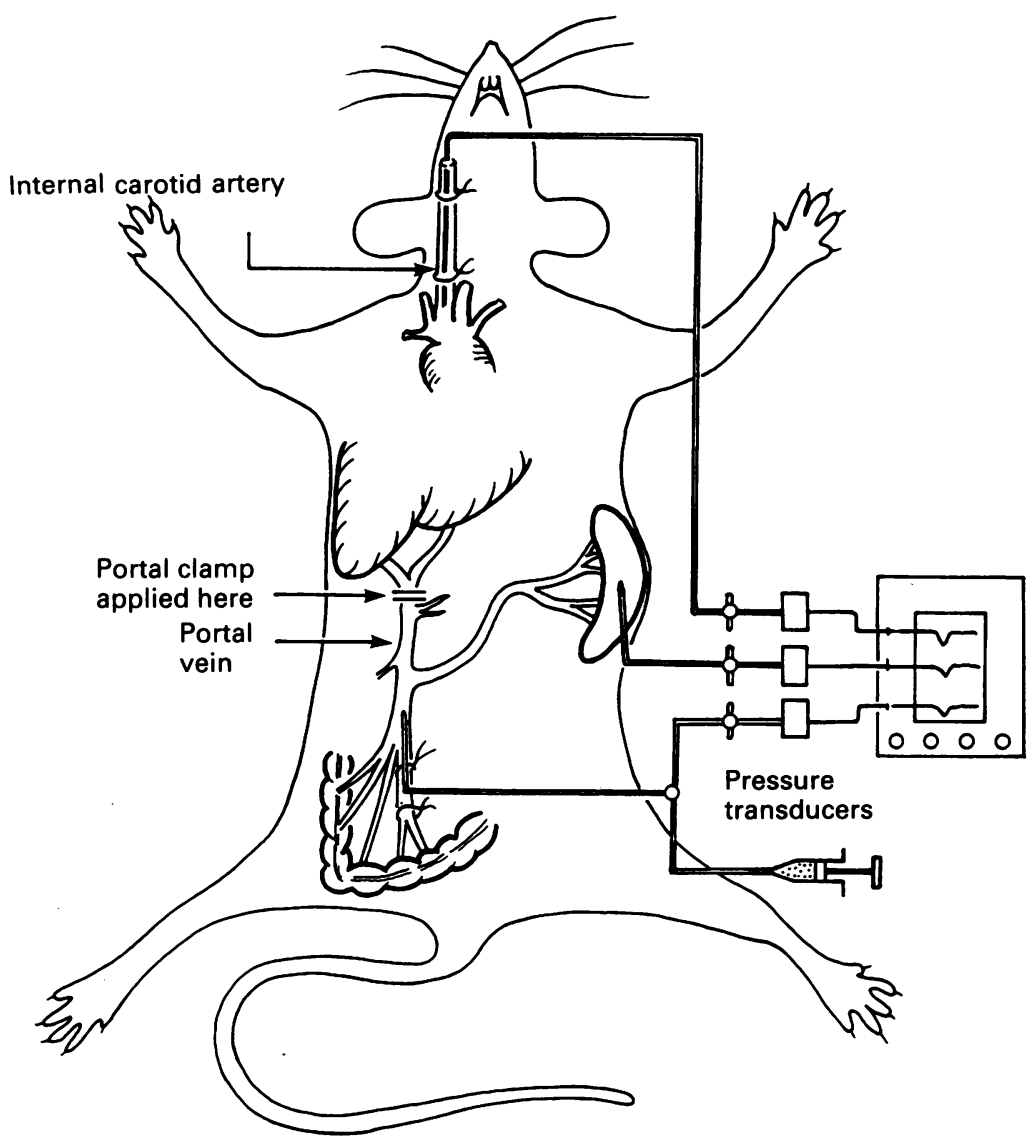

Figure 1: Schematic illustration of experimental preparation, showing sites of pressure measurement, microsphere injection, and portal vein occlusion.

applied to the portal vein at the liver hilum and pressure monitored (Fig 1). Animals were then rapidly killed with high dose intra-arterial barbiturate. Finally the liver, spleen, and lungs were removed for histological examination.

Statistics - statistical comparisons within and between groups were made using Student's paired and unpaired $t$ tests respectively, with $\mathrm{p}<0.05$ being regarded as statistically significant. Data are shown as mean (SEM).

\section{Results}

After two to five sequential injections, microsphere aliquots had begun to produce an increase in portal pressure to a peak that gradually declined to a new steady state within 30-60 seconds. Each subsequent aliquot raised the peak and steady state pressure further until a maximum was reached (after six to eight aliquots) (Fig 2). Once this maximum pressure was attained, no further pressure rise could be achieved even after further injections of large numbers of microspheres. No effect on arterial blood pressure was seen. Equivalent saline aliquots had no effect on portal or systemic pressures. Table II lists the basal, peak, and steady state pressures achieved after sequential injections of microspheres of increasing diameter. Each microsphere size produced a statistically significant increase in portal pressure from basal to peak $(8 \cdot 7-12.4 \mathrm{~mm} \mathrm{Hg}$ increase) and basal to steady state $(4 \cdot 0-5 \cdot 0 \mathrm{~mm}$ $\mathrm{Hg}$ increase) (Table II). There were no statistically significant differences between pressure increases produced by microspheres of different diameters. Splenic pulp pressures, though usually damped, closely paralleled those measured directly in the portal vein (Table II).

Hilar portal vein occlusion produced a dramatic rise in portal pressure from the new steady state to $57 \cdot 6(1 \cdot 2) \mathrm{mm} \mathrm{Hg}$ (Fig 3 ). Release of the occlusion was followed by a return to the previous hypertensive resting state. Histological examination of sections of the liver showed the presence of large numbers of microspheres. In particular, $15 \mu \mathrm{m}$ spheres were seen in the smallest portal radicles (Fig 4A), while $90 \mu \mathrm{m}$ spheres lodged in larger portal radicles (Fig 4B). No microspheres were seen in the spleen, but many spheres of all diameters were noted in the lungs (Fig 4C).

\section{Discussion}

The technique of directly introducing microspheres into the portal tract was developed in our laboratory to measure fractional distribution of splanchnic effluent after portal division. ${ }^{4}$ During those studies it was found that by introducing microspheres through a peripherally placed cannula (in the caecal vein) streaming would be avoided and mixing would be thorough. In those studies, in which low volumes of $15 \mu \mathrm{m}$ labelled microspheres were slowly infused manually, $95 \%$ lodged with an even distribution in the microvasculature of the liver. ${ }^{4}$ The same technique was utilised in setting up this model to achieve an even distribution of large numbers of microspheres within the liver parenchyma. Particular care was taken over the manual injection to avoid artificially high portal pressures, which might force microspheres into extrahepatic portal vein tributaries. The fact that synchronous splenic pressure monitoring in control animals showed no rise confirms that no such high pressures were produced.

Sequential aliquots of microspheres (in preference to single large bolus injections) were used
Figure 2: Example of portal vein $(P V)$ pressure recording obtained from a rat during intraportal microsphere injections.

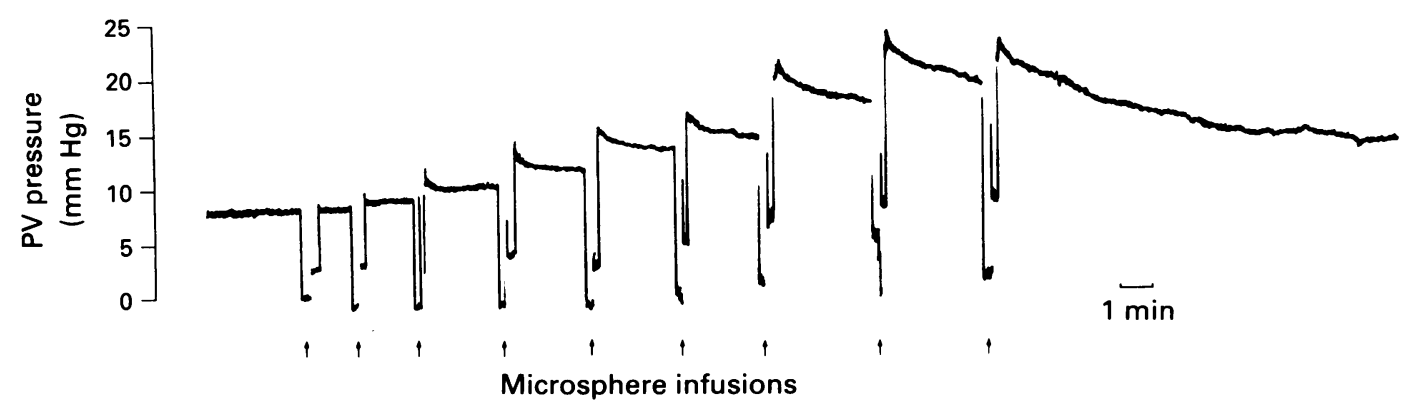


TABLE II Changes in portal pressure with microsphere injections

\begin{tabular}{|c|c|c|c|c|c|c|}
\hline \multirow{3}{*}{$\begin{array}{l}\text { Microsphere } \\
\text { size }(\mu m)\end{array}$} & \multicolumn{6}{|c|}{ Portal pressure $(\mathrm{mm} \mathrm{Hg}$ ) } \\
\hline & \multicolumn{3}{|c|}{ Direct recording } & \multicolumn{3}{|c|}{ Intrasplenic recording } \\
\hline & Basal & Peak & Steady state & Basal & Peak & Steady state \\
\hline $\begin{array}{l}15 \\
25 \\
50 \\
90\end{array}$ & $\begin{array}{r}10.1(0.6) \\
9.8(1.0) \\
9.3(0.3) \\
8.7(0.5)\end{array}$ & $\begin{array}{l}22.5(1.5) \\
18.5(1.9) \\
19.1(1.8) \\
18.8(1.8)\end{array}$ & $\begin{array}{l}15 \cdot 1(0.6) \\
13.9(1.0) \\
13.3(0.5) \\
13.4(0.9)\end{array}$ & $\begin{array}{c}10.4(0.6) \\
10.6(1.0) \\
9.1(0.5) \\
9.9(0.6)\end{array}$ & $\begin{array}{l}20 \cdot 5(1 \cdot 8) \\
19 \cdot 8(2 \cdot 1) \\
16 \cdot 5(2 \cdot 7) \\
17 \cdot 0(1 \cdot 7)\end{array}$ & $\begin{array}{l}14 \cdot 1(0 \cdot 5) \\
15 \cdot 6(1 \cdot 5) \\
12 \cdot 5(0 \cdot 9) \\
14 \cdot 3(1 \cdot 1)\end{array}$ \\
\hline
\end{tabular}

Data shown as mean (SEM).

Figure 3: Schematic illustration of typical venous traces from a rat during microsphere injections and subsequent portal vein occlusion. systemic arterial and portal

for three reasons: firstly, large volume injections were avoided; secondly, the minimum number of microspheres needed to produce hypertension was evaluated; and thirdly, intermittent small aliquot infusions permitted a more subtle insight into the haemodynamics of a slowly rising portal pressure (vide infra).

The anatomical basis for our model was that the intrahepatic portal tract branches into progressively smaller radicles until the sinusoids are reached. Classic morphology states that this branching is arranged in series, and our original work with radiolabelled spheres - where over $95 \%$ of spheres lodged in liver and negligible numbers passed through to lodge in lungs seemed to confirm this. If this is the case, then progressive occlusion of the intrahepatic radicles should produce a progressive rise in portal pressure. The extent of the portal hypertension acutely induced by microspheres $(13 \cdot 3-15 \cdot 1 \mathrm{~mm}$ $\mathrm{Hg}$ ) compares favourably with other models utilising partial portal vein ligation ${ }^{8-11}$ or cirrhosis. ${ }^{1012}$ Furthermore the hypertensive effect was consistently and reliably achieved in all animals.

Although microsphere diameter had little effect on the rise in portal pressure it was interesting to find that smaller numbers of the larger spheres were required. This would fit well with our original hypothesis of the way in which microspheres occlude the portal microvasculature within the liver. Larger spheres should lodge more proximally well before the sinusoidal

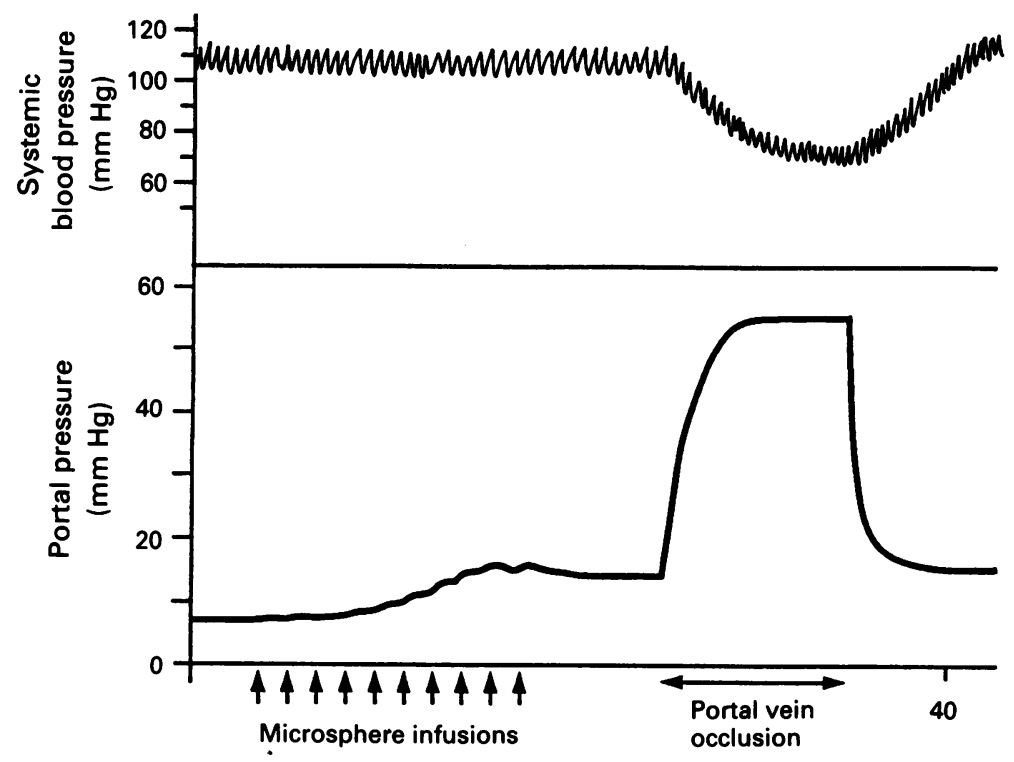

Time (min)
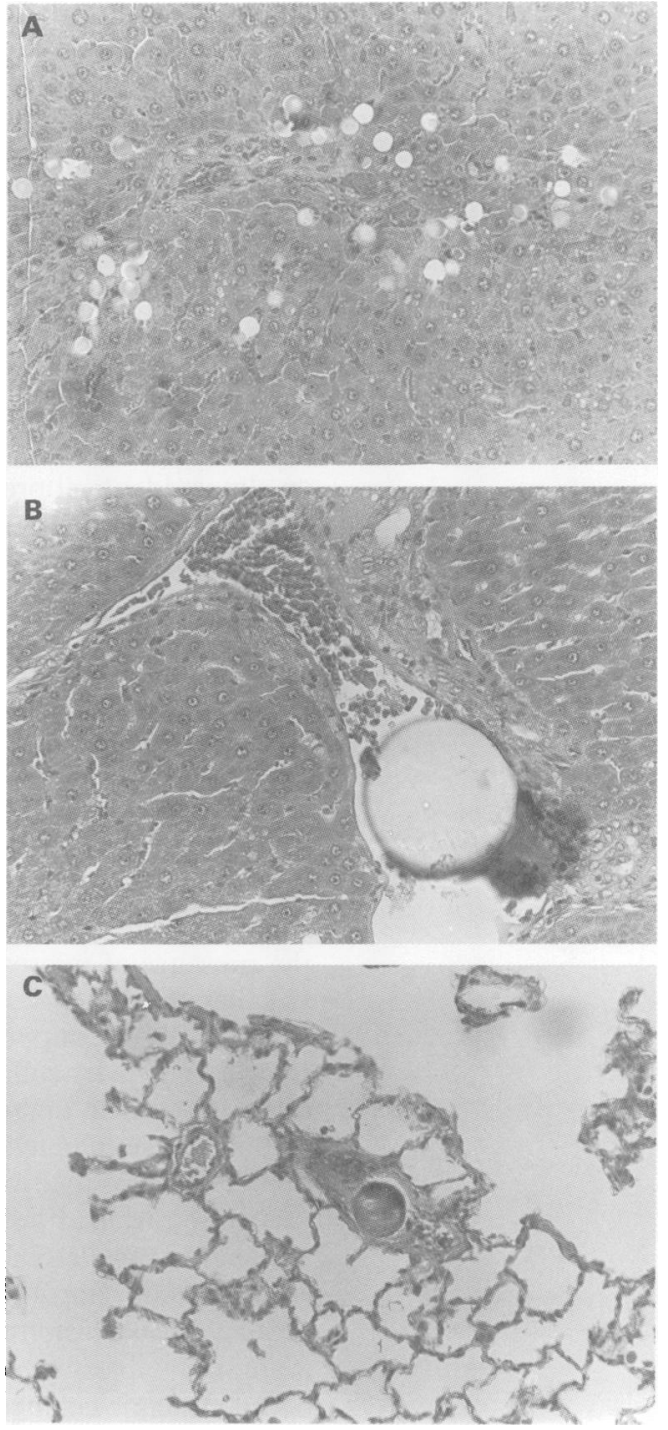

Figure 4: Histological sections (original magnification $\times 20$ ), showing $(A) 15 \mu \mathrm{m}$ sphere in liver, $(B) 90 \mu \mathrm{m}$ sphere in liver and $(C) 50 \mu m$ sphere in lung.

bed is reached and therefore smaller numbers produce an effect similar to that induced by a greater number of smaller spheres, which lodge most distally. Confirmation was achieved histologically, where $90 \mu \mathrm{m}$ microspheres were seen trapped in large portal radicles. By contrast, $15 \mu \mathrm{m}$ spheres were seen principally within the finest and most distal portal radicles.

The higher 'peak' pressures in comparison with the steady state pressure is probably an acute 'volume' effect. Even a volume of $0.2 \mathrm{ml}$ injected slowly into a portal vein that is partially obstructed has an effect, which is seen most obviously in the portal vein cannula recording and to a lesser extent in the splenic pulp. As the portal flow through the liver becomes progressively obstructed, the effect becomes more obvious - with peak pressures being 4-7 $\mathrm{mm} \mathrm{Hg}$ higher than the subsequent steady state measurement. In fact, small volume saline injections given at this stage (that is, after microspheres), produced small but significant pressure increases and therefore this model of PHT, once established, seems to have a reduced portal tract 'capacitance'. As noted above, control intraportal saline injections given before micro- 
spheres have no effect on portal or splenic pressure.

Although the final portal pressure $(13 \cdot 3-15 \cdot 1$ $\mathrm{mm} \mathrm{Hg}$ ) produced acutely by microspheres compares favourably with the other portal hypertension models (and also a recent report from Japan ${ }^{13}$ using larger microspheres in rabbits), it is intriguing that much higher pressures are not attainable. In previous work in our laboratory, where the extrahepatic portal tract was totally occluded (by a clamp applied across the portal vein at the liver hilum), portal pressure reached a mean of $56.0 \mathrm{~mm} \mathrm{Hg},{ }^{14}$ and in the present study an almost identical pressure was found. Assuming an even distribution of spheres, it should be possible progressively to occlude all the intrahepatic radicles, at which stage the pressure should approximate to that achieved by total portal occlusion. The inability of microspheres to raise the pressure to such values seems to show that, as the pressure within the intrahepatic portal tract is raised, microspheres (as large as 90 $\mu \mathrm{m})$ bypass the hepatic microvascular bed into the general venous circulation. Although not precisely recorded by radiobiological techniques, we have confirmed that this occurs by showing the presence of microspheres in the pulmonary vascular bed.

Originally we supposed that, as the pressure rose, extrahepatic collaterals became patent and that subsequent microsphere infusions passed through these channels into the systemic circulation, bypassing the liver. Two factors challenge this supposition. Firstly, it is well known that extrahepatic collaterals take days rather than seconds to develop. ${ }^{61516}$ This explains why total acute portal occlusion at the liver hilum in the rat is not survivable in the absence of pre-established portal systemic collaterals. ${ }^{914}$ Secondly, we have shown in our own laboratory ${ }^{14}$ that if extrahepatic portal systemic shunts have been induced, total hilar occlusion of the portal vein results in a peak pressure of only $20-30 \mathrm{~mm} \mathrm{Hg}$. If it is proposed that microspheres bypass the liver by newly induced extrahepatic collaterals, then total portal occlusion should result in a considerably reduced pressure rise. This was not the case: peak pressures after portal occlusion in microsphere treated animals reached about $60 \mathrm{~mm} \mathrm{Hg}$, similar to that achieved in control animals with no extrahepatic shunts. It is therefore highly unlikely that shunting occurs by the extrahepatic route, and thus it must be concluded that intrahepatic channels provide the portal systemic conduit.

We therefore propose that intrahepatic portal systemic shunts exist in the normal rat liver. Previously these have been reported only in cirrhotic livers in rats ${ }^{17}$ and in humans. ${ }^{18}$ These intrahepatic shunts must be of large diameter $(>90 \mu \mathrm{m})$, 'open' rapidly in response to a raised portal pressure, and permit shunting of blood directly into the hepatic veins. This would explain why increasing numbers of microspheres have no further pressure raising effect, why microspheres can be found in large numbers in the pulmonary bed and why, when these intrahepatic shunts are excluded by prehepatic clamping, the portal pressure rises to a value usually only seen in the total absence of any collaterals.

One of the significant advantages of this microsphere model is that portal hypertension is induced acutely while sparing the normal liver architecture. We believe that this model is reliable, reproducible, and clinically more relevant than prehepatic occlusion models. We intend to extend our measurements to a longer term study after microsphere injection, and to use this model to study pharmacological control of the hypertensive portal tract and to confirm the existence of intrahepatic portal systemic shunts in the normal rat liver.

This work was funded by a grant from the Hammersmith and Queen Charlotte's Special Health Authority. We thank Miss Marhokh Nohadani for preparing the histological slides and Mrs Denise Ryan for typing the manuscript.

1 Reichen J. Liver function and pharmacological considerations in pathogenesis and treatment of portal hypertension. Hepatology 1990; 11: 1066-78.

2 Lebrec D, Poynard T, Hillon P, Benhamou J-P. Propranolol for prevention of recurrent gastrointestinal bleeding in patients with cirrhosis. A controlled study. $N$ Engl F Med patients with cirrhosis.

3 Navasa M, Chesta J, Bosch J, Rodes J. Reduction of portal pressure by isosorbide-5-mononitrate in patients with cirrhosis. Gastroenterology 1989; 96: 1110-8.

$4 \mathrm{Jaffe} \mathrm{V}$. Studies on isolated liver cell transplants in the rat spleen - the effects of portal perfusion and the development of hepatocytes within the pancreas [Thesis]. Cambridge: 1989.

5 Vorobioff J, Bredfeldt JE, Groszmann RJ. Hyperdynamic circulation in portal-hypertensive rat model: a primary factor for maintenance of chronic portal hypertension. Am F Physiol 1983; 244: G52-7.

6 Choikier M, Groszmann RJ. Measurement of portal-systemic shunting in the rat by using $\gamma$-labeled microspheres. Am f Physiol 1981; 240: G371-5.

7 Kretschmer GJ, Sutherland DER, Matas AJ, Payne WD, Najarian JS. Autotransplantation of pancreatic fragments to the portal vein and spleen of totally pancreatectomized dogs: a comparative evaluation. Ann Surg 1978; 187: 79-86.

8 Halvorsen JF, Myking AO. Prehepatic portal hypertension in the rat. Immediate and long-term effect on portal vein and aortic pressure of a graded portal vein stenosis, followed by occlusion of the portal vein and spleno-renal collaterals. Eur Surg Res 1979; 11: 89-98.

9 Bengmark S, Boriesson B, Olin T. Transposition of the spleen in rats with portal hypertension. BrF Surg 1976; 63: 268-71.

10 Groszmann RJ, Vorobioff J, Riley E. Splanchnic hemodynamics in portal-hypertensive rats: measurement with $\gamma$-labeled microspheres. Am f Physiol 1982; 242: G156-60.

11 Benoit JN, Womack WA, Korthuis RJ, Wilborn WH, Granger DN. Chronic portal hypertension: effects on gastric Granger DN. Chronic portal hypertension: effects on gastric

12 Lee SS, Girod C, Braillon A, Hadengue A, Lebrec D. Hemodynamic characterization of chronic bile duct-ligated rats: effect of pentobarbital sodium. Am $\mathcal{F}$ Physiol 1986; 251 : G176-80.

13 Komeichi H, Katsuta Y, Aramaki T, Okumura H. A new experimental animal model of portal hypertension. Intrahepatic portal obstruction by injecting DEAE-cross-linked dextran microspheres into the portal vein in the rabbit. Nippon Ika Daigaku Zasshi 1991; 58: 273-84.

14 Jaffe V, Mathie RT. Dynamic pressure measurements in the evaluation of a pancreaticolienal portal diversion model in the rat. Eur Surg Res 1990; 22: 263-9.

15 Sikuler E, Groszmann RJ. Interaction of flow and resistance in maintenance of portal hypertension in a rat model.

16 Jaffe V, Sandin B, Wilkins RA. Radiological changes following acute portal vein occlusion in the rat. Brf Radiol 1990; 63: acute port

17 Wood AJJ, Villeneuve JP, Branch RA, Rogers LW, Shand DG. Intact hepatocyte theory of impaired drug metabolism in experiment

18 Hoefs J, Sakimura I, Reynolds T. Direct measurement of intra-hepatic shunting by the portal vein injection of microspheres. Gastroenterology 1978; 75: 968. 